\title{
Throughput and Delay Bounds for Cognitive Transmissions
}

\author{
F. Borgonovo, M. Cesana, L. Fratta
}

\begin{abstract}
Cognitive networks are based on agile and opportunistic use of spectrum resources. This work focuses on those network scenarios where primary or licensed users coexist with secondary or unlicensed ones. Secondary users opportunistically access the shared resources whenever vacant, with the strict constraint of being invisible to primary users. We derive here analytical bounds on throughput and transmission delay of secondary users under different assumptions on secondary and primary users traffic statistics, and we comment on the use of the proposed models to dimension secondary transmissions.
\end{abstract}

\section{Introduction}

The world of wireless communications is nowadays facing a serious problem of spectrum shortage. Such problem is not only due to "real" limitations on the available bandwidth, but also (and mainly) to inefficient policies in spectrum management. Indeed, todays wireless networks are characterized by a fixed spectrum assignment policy, which often leads to waste large spectrum portions due to sporadic utilization by the licensed users [1]. This situation is driving the development of novel spectrum sharing paradigms, with huge interest of different actors: academia, standardization bodies, spectrum regulation bodies and manufacturers.

The enabling technology at the physical layer is constituted by Cognitive Radios devices with capabilities of sensing the spectrum and dynamically adapting their transmission parameters in an agile and opportunistic way [2, 3]. Such technology

\footnotetext{
The authors are with the:

Advanced Network Technologies Laboratory (ANT Lab),

Dipartimento di Elettronica e Informazione,

Politecnico di Milano,

Piazza L. da Vinci 32,

20133 Milan, Italy,

e-mail: \{borgonov, cesana, fratta\} @elet.polimi.it
}

Please use the following format when citing this chapter:

Borgonovo, F., Cesana, M., Fratta, T., 2008, in IFIP International Federation for Information Processing, Volume 265, Advanees in Ad IIoc Networking, eds. Cuenca, P., Guerrero C., Puigjaner, R., Serra, B., (Boston: Springer), pp. 179190. 
at the physical layer is consequently driving the development of a new networking paradigm, as well, which is often referred to as Cognitive Networking.

We consider in this paper a general cognitive scenario, where the end users are distinguished into primary and secondary ones. Primary users or transmissions are allowed to use exclusively the licensed portions of the spectrum, whereas secondary transmissions may occasionally access the licensed spectrum in an opportunistic way, whenever it is vacated by the primary users. Secondary users are therefore equipped with Cognitive tunable Radios and may share the primary band with the licensed users with the strict constraint not to harm primary ones.

On the secondary users'side, this calls for effective mechanisms to dynamically detect spectrum usage, identifying which portions of the spectrum are free for use at each time, to quickly reconfigure the radio interface to chase "spectrum holes", and finally, to effectively coordinate with other secondary users, resolving collisions and/or scheduling transmissions.

The aforementioned functionalities are referred to as the cognitive cycle [4], which involves spectrum sensing and spectrum decision, to effectively detect the presence of primary users, spectrum sharing and spectrum access, to effectively coordinate with other secondary users accessing the shared resources. Generally speaking, the achievable performance of cognitive transmissions depends on the efficiency of the protocols handling the aforementioned phases of the cognitive cycle (sensing, decision, sharing and access).

In this work, we aim to provide bounds on the throughput and delay performance of secondary users in the aforementioned cognitive scenario, when assuming an idealized cognitive cycle. Namely, we leverage queuing theory to derive closed form expressions for the throughput and delay of secondary users in case of perfect sensing, and spectrum access; moreover, we comment on the impact of the traffic characteristics (packet dimensions, primary traffic load, traffic statistics, etc.) on the derived bounds.

The paper is organized as follows: in Section 2, we give an overview of the literature in the field of cognitive networks modelling. In Section 3, we derive throughput bounds for secondary transmissions, whereas in Section 4 we show how to use the derived throughput bounds to optimize the packet length of secondary transmissions. Delay bounds are derived in Section 5, whereas Section 6 concludes the paper by commenting on its contributions, and proposing directions for future work.

\section{Related Work}

Since Mitola's PhD dissertation in 2000 [2], the research in the field of cognitive networks has been extremely lively and rich. Broadly speaking, the research production can be classified depending on the specific cognitive cycle problem addressed. References [5, 6] focus on spectrum sensing issues. In [5], the authors propose a collaborative sensing approach which allows secondary users to share sensing information to increase the overall sensing reliability; on the other hand, Kim and Shin 
study in [6] proactive and reactive sensing mechanism and propose an algorithm to dynamically decide the optimal sensing approach.

The issues related to spectrum decision, access and management are addressed in [7, 8, 9]. In [7], the authors propose a distributed Medium Access Control (MAC) protocol able to handle spectrum sensing, decision and access at the same time. Decision theory is used to design and optimize the protocol parameters, with particular attention to spectrum prediction functionalities. In [8], Jia et al. design a MAC protocol which accounts for hardware constraints (single transceiver, sensing overhead), whereas Su and Zhang [9] leverage a cross-layering approach to implement a MAC based on cooperative sensing among secondary users.

The aforementioned pieces of work are targeted to the design and optimization either of physical layer sensing functionalities, or medium access control ones. In either cases, the focus is on single hop cognitive links. On the other side, also cognitive multi-hop wireless networks are recently attracting considerable attention [10]. In [11], Chowdhuri and Akyildiz extend the design of spectrum sensing and management techniques to the environment of wireless mesh cognitive networks, whereas references $[12,13,14]$ address the problem of routing optimization in multi-hop cognitive environments.

In this paper, we are not concerned with the design of specific protocols for cognitive networks, but rather we aim at developing a modelling framework to evaluate the performance of cognitive scenarios, eventually providing guidelines for the optimization of the implemented protocols.

Generally speaking, the evaluation of cognitive networks is a new and intriguing field of research since many variables and parameters concur to its definition. In this field, reference [15] proposes a dirty paper coding technique to maximize the throughput of secondary users with selfish behavior. Jovicic and Viswanath address in [16] the selfless case where secondary users cooperate in relaying primary messages, and derive information-theoretic bounds on the achievable rates. An information theoretic approach is used also in [17] to find throughput bounds when cooperation among primary and secondary users is not possible.

Besides information theory, game theory is also a widely used analytical tool to characterize the cooperative/non-cooperative behavior of secondary users in dynamic environments (e.g., [18]). See [19] for a nice review of the field.

In general, the common approach to assess the capacity of cognitive transmissions resorts to information theory or game theory, and neglects (primary and secondary) traffic dynamics; on the other hand, we are able to capture traffic statistics by leveraging queuing theory in the throughput and delay analysis. Queuing theory is also applied to Cognitive Radio environments in [20], where the authors study the stability of the queues of two single-link users (one primary user and one secondary user), under different assumptions on cooperation and sensing reliability. 


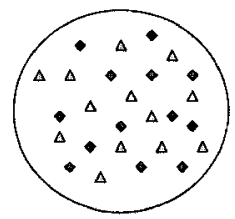

a)

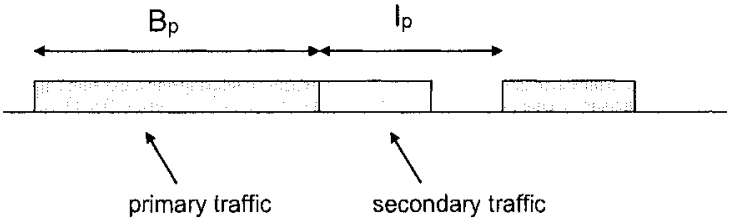

b)

Fig. 1 Cognitive network scenario with primary (triangles) and secondary (diamonds) users (a), a sample of the channel occupation out of primary and secondary transmissions (b).

\section{Throughput Bounds}

We consider a network scenario where sets of primary and secondary users potentially share a common radio resource (see Figure 1.a), and secondary users completely avoid to generate interference on primary users by accessing unoccupied spectrum portions, only.

Since we are mainly interested in studying the impact on throughput and delay of cross-traffic interference (primary vs secondary), we start off from the following simplifying assumptions, which refer to an ideal system and are adopted to obtain bounds to the performance of a real system.

- primary/secondary users co-location; primary and secondary transmissions sharing the same radio resources make up a fully connected cluster, that is, each transmissions is received by all the users in the cluster (either primary or secondary ones);

- perfect intra-class scheduling; collisions among users of the same type are avoided;

- perfect sensing; secondary transmissions have perfect sensing capabilities of primary traffic, that is, no sensing errors (detections, false positives) occur;

- ideal collision detection: secondary ongoing transmissions leave the channel as soon as a primary one is detected without causing any interference to the primary incoming transmissions;

A first rough bound on the throughput can be obtained observing that the overall system (primary+secondary transmissions) can be modelled as an $\mathrm{G} / \mathrm{G} / 1$ queue with prioritized users with preemption-resume of low priority users [21]. Let $\mu_{p}$ and $\mu_{s}$ be the average service frequency experienced by primary and secondary users, respectively; the average achievable throughput values of primary and secondary users $\left(\lambda_{p}, \lambda_{s}\right)$ are given by:

$$
\lambda_{p} \leq \mu_{p}, \lambda_{s} \leq \mu_{s}\left(1-\rho_{p}\right),
$$

where $\rho_{p}=\frac{\lambda_{p}}{\mu_{p}}$. 
Proof of first part of Eq. (1) is straightforward since the primary traffic is not affected by secondary one. To prove the second part, we leverage the concept of secondary users extended service time (or completion time according to the jargon of [22]), $C_{s}$, defined as the time it takes for a secondary packet to go through ${ }^{1}$. The calculation of the extended service time comes easily from the observation that each secondary transmission takes its own service time plus the service time of all the preempting primary transmissions entering during the extended service time itself, that is,

$$
E\left[C_{s}\right]=E\left[X_{s}\right]+\frac{\lambda_{p}}{\mu_{p}} E\left[C_{S}\right],
$$

being $X_{s}$ the random variable representing the secondary users transmission time. The average throughput of secondary users $\left(\lambda_{s}\right)$ is upper bounded by the reciprocal of the extended service time $\left(E\left[C_{s}\right]\right)$.

$$
\lambda_{s} \leq 1 / E\left[C_{s}\right]
$$

Substituting Eq. (3) in (2) and solving in $\lambda_{s}$ we obtain the second part of Eq. (1).

The throughput bound provided by Eq. (1) is over-optimistic, since it is based on the simplifying assumption that the secondary users' packets preempted by primary users halt the service until channel is free, at which time the secondary transmission is continued from the time at which it was interrupted ${ }^{2}$.

In real wireless communication systems, work conserving (preemption-resume) strategy is not feasible, since each transmitted packet must carry signalling information (e.g. bits for the Cyclic Redundancy Check, physical layer preambles, MAC addresses etc.); consequently, whenever a transmission is aborted, the corresponding packet must be entirely retransmitted, eventually repeating in the retransmission the very same signalling information carried by the original transmission.

If the resume assumption is dropped and preempted packets must be fully retransmitted, the analysis of the secondary users extended service time is more elaborated, since the transmission of secondary packets is no longer a work-conserving process. Let's suppose that the traffic (arrival and service processes) generated by primary users are statistically known. Figure 1.b shows a realization of the traffic process associated to primary transmissions, where r.v. $B_{p}$ and $I_{p}$ represent the busy period and the idle period of the primary traffic, respectively. Secondary traffic can only exploit the idle periods of primary sources to go through.

Assuming saturation condition for secondary transmissions, the secondary throughput is given by $\lambda_{s}^{*}=1 / E\left[C_{s}^{*}\right]$, where $E\left[C_{s}^{*}\right]$ is the average extended service time of a secondary user, which can be expressed as follows, according to the total probabilities theorem:

$$
E\left[C_{s}^{*}\right]=\int_{0}^{+\infty} E\left[C_{s}^{*} / X_{s}=x\right] f_{X_{s}}(x) d x
$$

\footnotetext{
${ }^{1}$ including all the time wasted due to preemption of primary users

2 work-conserving preemption strategy
} 
The extended service time depends on whether the accessing secondary transmission fits into the corresponding idle period of the primary traffic. The preemption/nonpreemption probabilities $q$ and $p$ of a secondary packet of duration $x$ are given by:

$$
\begin{gathered}
p=P\left(I_{p}>X_{s} / X_{s}=x\right)=1-F_{I_{p}}(x) \\
q=1-p,
\end{gathered}
$$

where $F_{I_{p}}()$ is the c.d.f. of r.v. $I_{p}$. Moreover, the average duration of primary traffic idle period where preemption happens can be written as:

$$
E\left[I_{p} / I_{p} \leq x\right]=\int_{0}^{x} t \frac{f_{l_{p}}(t)}{F_{I_{p}}(t)} d t
$$

In case the primary users idle period $\left(I_{p}\right)$ is exponentially distributed, Eq. (6) leads to:

$$
E\left[I_{p} / I_{p} \leq x\right]=\frac{1}{\lambda_{p}}-x \frac{p}{q}
$$

The extended service time of Eq. (4) conditioned to $X_{s}=x$ can be written as:

$$
\begin{aligned}
& E\left[C_{s}^{*} / X_{s}=x\right]= \\
= & x+\sum_{i=1}^{+\infty}(i-1) E\left[B_{p} / I_{p} \leq x\right] p q^{i-1}+ \\
& +\sum_{i=1}^{+\infty}(i-1) E\left[I_{p} / I_{p} \leq x\right] p q^{i-1} \\
= & x+\left(E\left[B_{p} / I_{p} \leq x\right]+E\left[I_{p} / I_{p} \leq x\right]\right)\left(\frac{1-p}{p}\right)
\end{aligned}
$$

The average extended service time and (consequently the throughput) of secondary transmissions is then obtained by substituting Eq. (6) into Eq. (8), and expressing the average duration of primary traffic busy period as $1 /\left(\mu_{p}\left(1-\rho_{p}\right)\right)$ in Eq. (4). The throughput for secondary users when secondary user service time is exponentially, deterministically and uniformly distributed with common average $1 / \mu_{s}$ is given by:

$$
\begin{gathered}
\lambda_{s}^{e x p}=\left(1-\rho_{p}\right)\left(\mu_{s}-\lambda_{p}\right) \quad \lambda_{s}^{d e t}=\frac{\lambda_{p}\left(1-\rho_{p}\right) e^{-\lambda_{p} / \mu_{s}}}{1-e^{-\lambda_{p} / \mu_{s}}} \\
\lambda_{s}^{\text {unif }}=\frac{2 \lambda_{p}^{2}\left(1-\rho_{p}\right)}{\mu_{s} e^{2 \lambda_{p} / \mu_{s}}-2 \lambda_{p}-\mu_{s}}
\end{gathered}
$$

Figure 2 shows the secondary users traffic versus the primary users traffic for different values of secondary users service time, in case of exponentially distributed secondary and primary users service time. From the results in Fig 2, one can observe that, as expected, the difference between the preemption resume and preemption repeat models increases as the ratio between primary users service time and secondary ones decreases, i.e., the probability of preemption increases. 


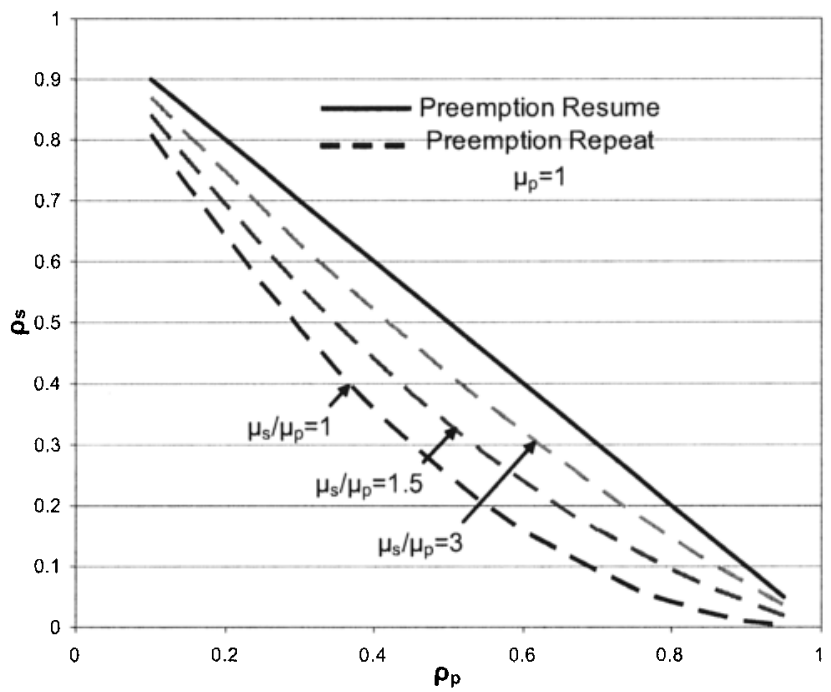

Fig. 2 Secondary traffic $\left(\rho_{s}\right)$ versus primary traffic $\left(\rho_{p}\right)$. Primary and secondary users service time with negative exponential distributions.

\section{Packet Length Optimization for Secondary Transmissions}

The analysis proposed in the previous section can be leveraged to select the packet length of secondary transmissions in order to optimize their throughput. Note that the throughput values obtained in Section 3 refer to the gross throughput, including physical and MAC headers. To measure the nominal throughput relevant to secondary users, let us define the average payload length (or duration ${ }^{3}$ ) of secondary packets,$\overline{L_{s}}$, and the header length (or duration), $h_{s}$. We observe that while $h_{s}$ is associated to specific communication protocols (PHY/MAC/L3 headers), the value of $\overline{L_{S}}$ depends on the specific application running at the secondary users.

Furthermore, let $C$ be the channel capacity shared by primary and secondary transmissions, $\overline{L_{p}}$ the average primary packet length, and $\mu_{s}=C / \overline{L_{s}}$ and $C / \mu_{p}=\overline{L_{p}}$ the average frequency of service for primary and secondary packets, respectively. The nominal throughput of secondary transmissions. in the case of work-conserving service model is given by: ${ }^{4}$ :

$$
\lambda_{s}<\left(1-\rho_{p}\right) \frac{\overline{L_{s}}}{\frac{h_{s}}{C}+\frac{\overline{L_{s}}}{C}},
$$

\footnotetext{
${ }^{3}$ if normalized to the channel bandwidth

${ }^{4}$ Calculation details are skipped since calculation comes directly from the analysis proposed in the previous section.
} 
whereas, in case of preemption-repeat of secondary transmissions we have:

$$
\lambda_{s}<\frac{\lambda_{p}\left(1-\rho_{p}\right)\left(\mu_{s}-\lambda_{p}\right)}{\mu_{s} e^{\lambda_{p} h_{s} / C}-\mu_{s}+\lambda_{p}} \bar{L}_{s},
$$

Figure 3 reports the secondary users throughput normalized on the channel capacity $(C)$ versus the average payload length of secondary packets $\left(\bar{L}_{s}\right)$, for different values of primary traffic $\left(\rho_{p}\right)$. The curves in the figure have been obtained considering the set of parameters $C=36 \mathrm{Mb} / \mathrm{s}, \overline{L_{p}}=500$ bytes, and an header dimension $h_{s}=40$ bytes. As clear form the figure, an optimum value of the secondary packets payload length does exist, coming from the trade off between transmission efficiency (large payload) and preemption probability (small packets).

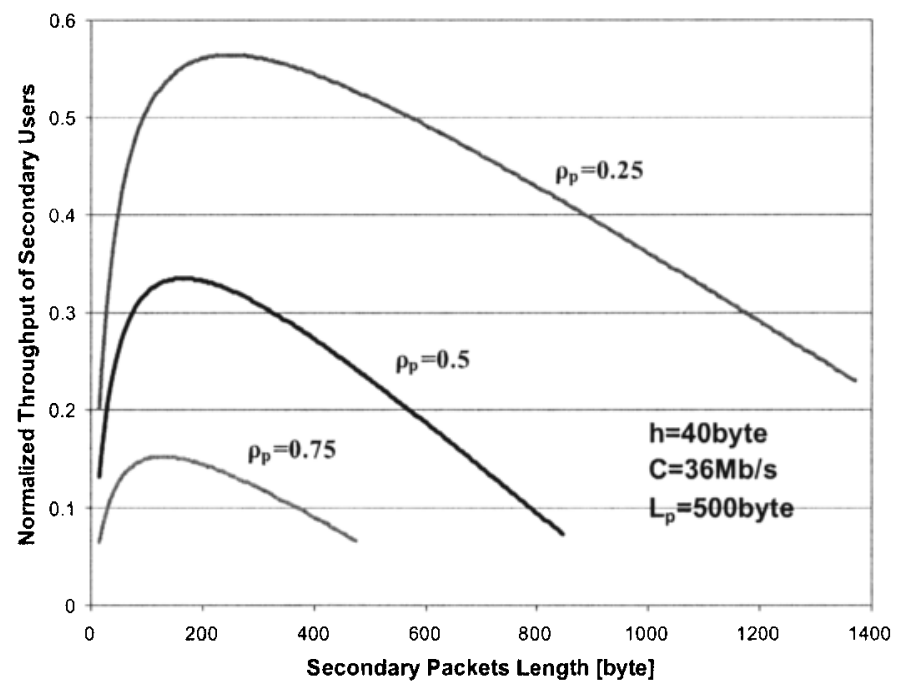

Fig. 3 Normalized Throughput of Secondary transmissions versus the average payload length $\bar{L}_{5}$; $C=36 \mathrm{Mb} / \mathrm{s}, L_{p}=500$ bytes, and $h_{s}=40$ bytes.

Such optimum value, $L_{s}^{\overline{o p t}}$, is formally characterized by the following equation:

$$
L_{s}^{\bar{p} p t}=\operatorname{argmax}_{\overline{L_{s}}} \lambda_{s}
$$

Figure 4 shows the behavior of $L_{s}^{\overline{p p t}}$ when varying the traffic of the primary users for the same parameter setting as the one of Figure 3. As clear from the figure, the optimal payload dimension for secondary transmissions decreases when increasing the primary traffic intensity $\left(\rho_{p}\right)$ and tends to level off for high values of it. 


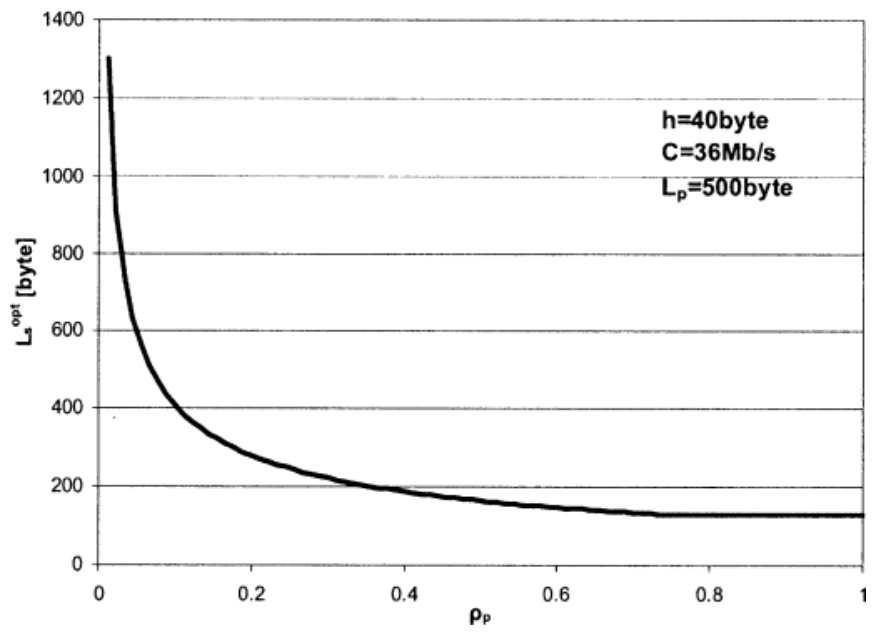

Fig. 4 Optimal value of the secondary transmissions payload length $L_{s}^{\overline{o p t}} ; C=36 \mathrm{Mb} / \mathrm{s}, \overline{L_{p}}=500$ bytes, and $h_{s}=40$ bytes.

\section{Delay Analysis}

The system delay defined as the total time spent in the system by a secondary packet is also an important performance figure to evaluate cognitive transmissions. To this end, we derive hereafter closed form expressions for the system delay distinguishing again between the two cases of preemption resume and preemption repeat for the secondary users transmissions.

In case of preemption resume and assuming Poisson statistics for primary and secondary transmissions, the system can be modelled as an M/G/1 queue with twoclasses priority users and preemption. Thus, the average waiting times of primary $\left(E\left[V_{p}\right]\right)$ and secondary $\left(E\left[V_{s}\right]\right)$ packets can be written as $[23]$ :

$$
\begin{gathered}
E\left[V_{p}\right]=m_{X_{p}}+\frac{\rho_{p}}{1-\rho_{p}} E\left[Z_{p}\right] \\
E\left[V_{s}\right]=\frac{m_{X_{s}}}{1-\rho_{p}}+\frac{\sigma_{p}}{\left(1-\sigma_{p}\right)\left(1-\rho_{p}\right)} \sum_{i \in\{s, p\}} \frac{\rho_{i}}{\sigma_{p}} E\left[Z_{i}\right]
\end{gathered}
$$

In case of preemption-repeat, we observe that the imbedded process of secondary transmissions can be modelled as an $\mathrm{M} / \mathrm{G} / 1$ queue with service time $C_{s}$. Consequently, the average waiting time of secondary packets can be expressed using the Pollaczek-Khintchine result [23]:

$$
E\left[V_{s}\right]=\frac{\rho_{s} E\left[Z_{s}\right]}{1-\rho_{s}}+E\left[C_{s}\right]
$$


where $E\left[Z_{s}\right]$ is the residual service time seen by secondary packet entering the system. According to the renewal theory, we can write:

$$
E\left[Z_{s}\right]=\frac{E\left[C_{s}\right]}{2}+\frac{\operatorname{Var}\left[C_{s}\right]}{2 E\left[C_{s}\right]} .
$$

$E\left[C_{s}\right]$ is given by Eq. (4), whereas a closed formula for $E\left[C_{s}^{2}\right]$ is given by [21]:

$$
\begin{gathered}
E\left[C_{s}^{2}\right]=2\left(E\left[B_{p}\right]+E\left[I_{p}\right]\right)^{2}\left[E\left(e^{\lambda_{p} X_{p}}-1\right)^{2}\right]+ \\
\left(E\left[B_{p}^{2}\right]+2 E\left[B_{p}\right] / \lambda_{p}+2 / \lambda_{p}^{2}\right)\left(E\left[e^{\lambda_{p} X_{p}}\right]-1\right) \\
-2\left(E\left[B_{p}\right]+1 / \lambda_{p}\right) E\left[X_{p} e^{\lambda_{p} X_{p}}\right]
\end{gathered}
$$

All the terms in the previous equation are known except for the second moment of the busy period of primary traffic, $E\left[B_{p}^{2}\right]$, which however, can be expressed as [23]:

$$
E\left[B_{p}^{2}\right]=\frac{E\left[X_{p}^{2}\right]}{\left(1-\rho_{p}\right)^{3}}
$$

By substituting Eq. (16) into Eq. (15) we get $E\left[Z_{s}\right]$, which inserted into Eq. (14), leads to the closed form expression for the secondary packets waiting time.

Figure 5 reports the average time spent in the system by secondary packets normalized to the average service time, when varying the traffic intensity of primary and secondary users. From the figure, the total time spent in the system by secondary packets increases when increasing the traffic intensity of both primary and secondary users. In the former case, the delay increase is mainly due to higher preemption probability and longer busy periods of primary users, whereas, in the latter case, the queuing delay within secondary users'queues is the main contribution to the overall delay.

\section{Concluding Remarks}

This paper leverages queuing theory to provide upper bounds to the throughput/delay performance of cognitive scenarios where primary licensed users coexist with secondary opportunistic ones. Namely, we derived closed form expressions for the achievable throughput of secondary users and the corresponding delay performances, and we discussed on the quality of the proposed bounds under different traffic conditions. Finally, we have shown how the throughput model can be used to optimize the payload length of secondary transmissions.

The utility of the proposed bounds on throughput and delay is twofold:

- Performance Evaluation; the models can be obviously adopted to evaluate and predict the performance of cognitive scenarios, providing a benchmark to evaluate the efficiency of MAC protocols and cooperative sensing approaches for cognitive radio 


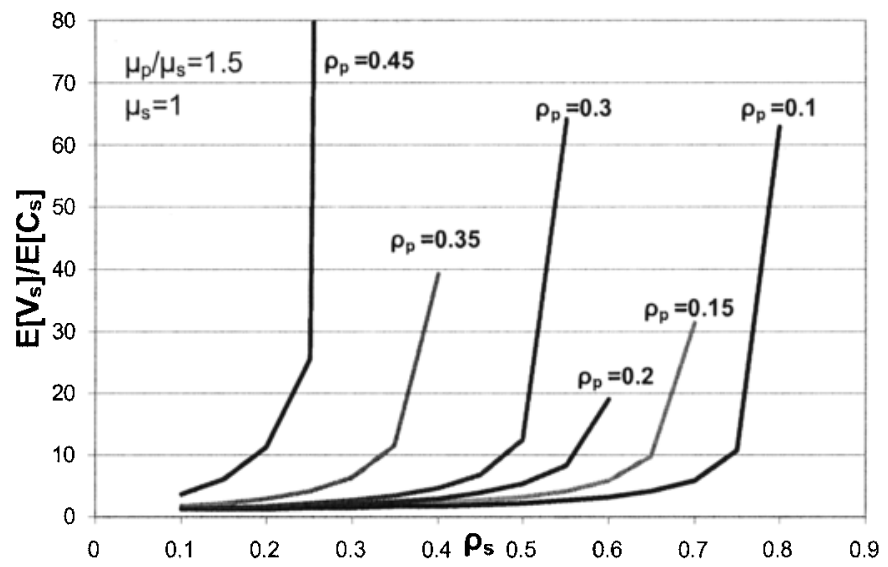

Fig. 5 Average Time spent in the system by secondary packets normalized to the average extended service time; $C=36 \mathrm{Mb} / \mathrm{s}, \overline{L_{p}}=500$ bytes, and $h_{s}=40$ bytes.

- Performance Optimization and dimensioning; the model has clearly shown that the performance of secondary transmissions (throughput and delays) is favored by the knowledge of primary traffic statistics $\left(\rho_{p}\right)$; therefore, the proposed model for the calculation of the nominal throughput and delay can be combined with techniques to estimate primary traffic statistics to design dynamic and adaptive dimensioning tools for secondary transmissions.

Natural follow ups of this work include the modelling of non-perfect sensing and collisions detection schemes, and the extension to partially connected scenarios where the channel statistics of the primary transmissions are spatially different throughout the cognitive network scenario.

\section{References}

1. Fereral Communications Commission, "Spectrum policy task force report," FCC 02-155, Nov. 2002.

2. J. Mitola, "Cognitive Radio: An Integrated Agent Architecture for Software Defined Radio", PhD Dissertation, KTH, Stockholm, Sweden, Dec. 2000.

3. S. Haykin, Cognitive Radio: Brain-Empowered Wireless Communications, IEEE JSAC, Vol. 23, No. 2, February 2005, pp. 201-220.

4. I.F. Akyildiz, W.Y. Lee, M.C. Vuran, S. Mohanty, "NeXt Generation/Dynamic Spectrum Access/Cognitive Radio Wireless Networks: A Survey", Computer Networks Journal, (Elsevier), September 2006.

5. A. Ghasemi, E.S. Sousa, "Collaborative spectrum sensing for opportunistic access in fading environment," in proc. of IEEE DySPAN 2005, November 2005, pp. 131-136. 
6. H. Kim, and K.G. Shin. "Adaptive MAC-layer Sensing of Spectrum Availability in Cognitive Radio Networks", Technical Report, CSE-TR-518-06, University of Michigan

7. Q. Zhao, L. Tong, A. Swami, and Y. Chen "Decentralized Cognitive MAC for Opportunistic Spectrum Access in Ad Hoc Networks: A POMDP Framework" IEEE JSAC, Vol. 25, No. 3. April 2007, pp. 589.600.

8. J. Jia, Q. Zhang, and X. Shen, "HC-MAC: A Hardware-constrained Cognitive MAC for Efficient Spectrum Management", IEEE JSAC, Vol. 26, No. 1, Jan. 2008, pp. 106-117.

9. H. Su, and X. Zhang, "Cross-Layer Based Opportunistic MAC Protocols for QoS Provisioning Over Cognitive Radio Wireless Networks", IEEE JSAC, Vol. 26, No. I, Jan. 2008, pp. 118-129.

10. T. Hou, Y. Shi, and H. D. Sherali, "Spectrum Sharing for Multi-hop Networking with Cognitive Radios", IEEE JSAC, Vol. 26, No. I, Jan. 2008, pp. 146-154.

11. K.R. Chowdhury, I.F. Akyildiz, "Cognitive Wireless Mesh Networks with Dynamic Spectrum Access", IEEE JSAC, Jan. 2008, Vol. 26, No. 1, pp. 168-181.

12. C. Xin, B. Xie, and C.C. Shen, "A novel layered graph model for topology formation and routing in dynamic spectrum access networks", in proc. of IEEE DySPAN 2005, pp. 308-317.

13. G. Cheng, W. Liu, Y. Li, W. Cheng, "Joint On-Demand Routing and Spectrum Assignment in Cognitive Radio Networks", in proc. of IEEE ICC 2007, pp. 6499-6503.

14. M. Ma, D.H.K. Tsang, "Joint Spectrum Sharing and Fair Routing in Cognitive Radio Networks", in proc. of IEEE CCNC 2008, pp. 978-982.

15. N. Devroye, P. Mitran and V. Tarokh, "Achievable Rates in Cognitive Radio Channels", IEEE Trans. Info. Theory, Vol. 52, May 2006, pp. 1813-1827.

16. A. Jovicic and P. Viswanath, "Cognitive Radio: An Information-Theoretic Perspective", IEEE International Symposium on Information Theory, July 2006, pp. 2413-2417.

17. S.A. Jafar and S. Srinivasa, "Capacity Limits of Cognitive Radio with Distributed and Dynamic Spectral Activity", IEEE JSAC, Vol. 25, No. 3, Apr. 2007, pp. 529-537.

18. O. Simeone, I. Stanojev, S. Savazzi, Y. Bar-Ness, U. Spagnolini, and R. Pickholtz, "Spectrum Leasing to Cooperating Secondary ad hoc Networks", IEEE JSAC, Vol. 26, No. l, Jan. 2008, pp. 203-213.

19. Z. Ji, and K.J. Ray liu, "Dynamic Spectrum Sharing: A Game Theoretical Overview", IEEE Comm. Mag., May 2007, vol. 5, No. 5, pp. 88-94.

20. O. Simeone, Y. Bar-Ness and U. Spagnolini, "Stable throughput of cognitive radios with and without relaying capability", IEEE Trans. Commun., Vol. 55, No. 12, Dec. 2007, pp. 2351-2360.

21. D.P. Graver, "A Waiting Line with Interrupted Services, Including Priorities", Journal of the Royal Statistical Society, 1962, Vol. 24, No. 1, pp. 73-90.

22. R.W. Wolff,"Work Conserving Priorities", Journal of Applied Probabilities, August 1970, Vol. 7, No. 2, pp. 327-337.

23. L. Kleinrock, "Queueing Systems: Computer Applications - Vol. 2", Wiley, May 1976. 